\title{
E. G. Kalnins
}

Mathematics Department, University of Waikato, Hamilton, New Zealand

Willard Miller, Jr.

School of Mathematics, University of Minnesota, Minneapolis, Minnesota 55455

(Received 28 November 1984; accepted for publication 8 February 1985)

\begin{abstract}
We show that additive separation of variables for linear homogeneous equations of all orders is characterized by differential-Stäckel matrices, generalizations of the classical Stäckel matrices used for multiplicative separation of (second-order) Schrödinger equations and additive separation of Hamilton-Jacobi equations. We work out the principal properties of these matrices and demonstrate that even for second-order Laplace equations additive separation may occur when multiplicative separation does not.
\end{abstract}

\section{INTRODUCTION}

Our motivation for this study of additive separation of variables for linear differential equations was the following example in Ref. 1:

$$
\left(x_{1}+x_{2}\right)\left(\partial_{11} u+\partial_{22} u\right)-2\left(\partial_{1} u+\partial_{2} u\right)=E .
$$

This equation admits a five-parameter separable solution in the coordinates $x_{1}, x_{2}$ :

$$
\begin{aligned}
u= & \left(\alpha x_{1}^{3}+\beta x_{1}^{2}+\gamma x_{1}-\frac{1}{2} E x_{1}\right) \\
& +\left(-\alpha x_{2}^{3}+\beta x_{2}^{2}-\gamma x_{2}+\delta\right) .
\end{aligned}
$$

The mechanism of separation was puzzling to us until we realized that the appropriate separation equations are

$$
\begin{array}{lllll}
\partial_{1} u & +E / 2 & -\gamma & -2 \beta x_{1} & -3 \alpha x_{1}^{2}=0, \\
\partial_{11} u & & & -2 \beta & -6 \alpha x_{1}=0, \\
\partial_{2} u & & +\gamma & -2 \beta x_{2} & +3 \alpha x_{2}^{2}=0, \\
\partial_{22} u & & & -2 \beta & +6 \alpha x_{2}=0 .
\end{array}
$$

The associated "Stäckel matrix" responsible for the separation is

$$
\left[\begin{array}{cccc}
\frac{1}{2} & -1 & -2 x_{1} & -3 x_{1}^{2} \\
0 & 0 & -2 & -6 x_{1} \\
0 & 1 & -2 x_{2} & 3 x_{2}^{2} \\
0 & 0 & -2 & 6 x_{2}
\end{array}\right] .
$$

This is not a true Stäckel matrix since more than one row depends on a given variable $x_{I}$ (Refs. 2 and 3). Moreover, the second and fourth rows are the derivatives of the first and third rows, respectively. It is a nontrivial example of a differential-Stäckel matrix.

In this paper we show that the above example is not isolated. All additive separation of $n$ th-order linear differential equations $L=E$ or $L=0$ is associated with differentialStäckel matrices. In Sec. II we derive, in the form of a coupled system of nonlinear partial differential equations, necessary and sufficient conditions that a linear differential equation admits additive separation in a given coordinate system. In Sec. III we develop the principal properties of the matrices inverse to differential-Stäckel matrices, and in Sec. IV we find all solutions of the separability conditions and show that they correspond to differential-Stäckel matrices. Our method is an extension of Eisenhart's study of true Stäckel matrices. ${ }^{3}$ In Sec. V we comment on the relation between multiplicative separation and additive separation for Laplace equations on Riemannian manifolds, and we give an example to show that additive separation may occur for a Laplace equation in a given coordinate system even when multiplicative separation is absent.

All functions appearing in this paper are assumed to be locally real analytic.

\section{ADDITIVE SEPARABILITY FOR LINEAR DIFFERENTIAL EQUATIONS}

In Ref. 1 the authors introduced a general definition of additive separation of variables for a partial differential equation

$$
H\left(x_{I}, u, u_{I}, u_{I J}, \ldots\right)=E,
$$

in the coordinates $x_{1}, \ldots, x_{N}$. Here $u$ is the dependent variable, $u_{I}=\partial_{x_{I}} u, u_{I J}=\partial_{x_{I}} \partial_{x_{J}} u$, etc., and $E$ is a parameter. A separable solution of (2.1) is a solution of the form $u=\Sigma_{J=1}^{N} S^{(J)}\left(x_{J}, E\right)$. We briefly review this definition (a generalization of that of Levi-Civita ${ }^{4}$ and its simple consequences. (See Ref. 5 for a discussion of other definitions of separability.)

For convenience we suppose $H$ is a polynomial in the derivatives $u_{1}, u_{I J}, \ldots$. Furthermore, there is no loss of generality in setting all mixed partial derivatives identically equal to zero (since $u_{I J}=0$ for $I \neq J$ if $u$ is a separable solution) and writing (2.1) in the form

$$
H\left(x_{I}, u, u_{I}, u_{I I}, \ldots\right)=E .
$$

We introduce the new notation $u_{I, 1}=u_{I}$, $u_{I, i+1}=\partial_{x_{I}} u_{I, i}, i=1,2, \ldots$, and define $n_{I}$ to be the largest number $l$ such that $\partial u_{I, l} H=H_{u_{L I}} \equiv 0$. To avoid discussion of degenerate cases we require $n_{I}>0$ for $I=1, \ldots, N$.

Let the truncated differentiation operator $\widehat{D}_{I}$ be defined by

$$
\widehat{D}_{I}=\partial_{x_{I}}+u_{I, 1} \partial_{u}+\cdots+u_{I, n_{I}} \partial_{u_{i, n_{I}-1}} .
$$

In Ref. 1 we showed that every separable solution $u$ of $(2.2)$ satisfies the integrability conditions

$$
\begin{aligned}
H_{u_{i, n_{I}}} & H_{u_{J, n_{J}}}\left(\widehat{D}_{I} \widehat{D}_{J} H\right)+H_{u_{I, n_{I}} u_{J, n J}}\left(\widehat{D}_{I} H\right)\left(\widehat{D}_{J} H\right) \\
& -H_{u_{J, n J}}\left(\widehat{D}_{I} H\right)\left(\widehat{D}_{J} H_{u_{u_{I, n_{I}}}}\right)-H_{u_{I, n_{I}}}\left(\widehat{D}_{J} H\right)\left(\hat{D}_{I} H_{u_{J, n J}}\right) \\
= & 0, \quad 1 \leqslant I<J \leqslant N .
\end{aligned}
$$


If (2.3) is an identity in the dependent variables $u, u_{K, k}$ then we say that $\left\{x_{I}\right\}$ is a regular separable coordinate system. In this case the separable solutions involve $\Sigma_{J=1}^{N} n_{J}+1$ independent parameters; that is, at a fixed point $x^{0}$ the separable solutions are uniquely determined by prescribing $u\left(x^{0}\right)$ and the $\Sigma_{J=1}^{N} n_{J}$ derivatives $u_{i, i}\left(\mathbf{x}^{0}\right), 1 \leqslant I \leqslant N, 1 \leqslant i \leqslant n_{I}$. If the integrability conditions (2.3) do not hold identically then the separation is nonregular; separable solutions may exist but they will involve (strictly) fewer parameters than the regular case. In the following when we speak of variable separation we mean regular separation. [Note that multiplicative separation can easily be treated by the preceding definition since $v=\Pi_{J=1}^{N} T^{(J)}\left(x_{J}\right)$ is multiplicatively separable if and only if $u=\ln v$ is additively separable.]

For Laplacelike equations

$$
H\left(x_{I}, u, u_{I}, u_{I I}, \ldots\right)=0 \text {, }
$$

there is a minor modification of the integrability conditions. Denoting by $F_{I J}$ the left-hand side of Eqs. (2.3) we can state the integrability conditions for (2.4) in the form

$$
F_{I J}=P_{I, J} H, \quad 1 \leqslant I<J<N,
$$

where $P_{I, J}\left(x_{K}, u, u_{K, k}\right)$ are polynomials in $u_{K, k}$. If $(2.5)$ is satisfied identically in the dependent variables $u, u_{J, j}$ we say that $\left\{x_{K}\right\}$ is a regular separable coordinate system. In this case the separable solutions depend on $\Sigma_{J=1}^{N} n_{J}$ independent parameters. For nonregular separation the separable solutions depend on fewer parameters.

Now we will apply these criteria to determine additive separability conditions for the linear equations

$$
L=E
$$

and

$$
L=0 \text {, }
$$

where

$$
L=\sum_{J=1}^{N} \sum_{j=1}^{n_{J}} H_{\langle J, j)}(x) u_{J, j}, \quad H_{\left(J, n_{j}\right)} \neq 0 .
$$

Introducing the abbreviation $H_{J} \equiv H_{\left(J, n_{J}\right)}$ we can write the integrability conditions (2.3) for $L=E$ in the form

$$
\widehat{D}_{I} \widehat{D}_{J} L-\widehat{D}_{I} L \partial_{J} \ln H_{I}-\widehat{D}_{J} L \partial_{I} \ln H_{J}=0, \quad I \neq J,
$$

where $\partial_{I}=\partial_{x_{I}}$. Equating to zero the coefficients of the derivatives $u_{J, j}$ on the left-hand side of (2.9) we obtain the following necessary and sufficient conditions for regular separation:

$$
\begin{aligned}
& \partial_{I J} H_{(P, p)}-\partial_{I} H_{(P, p)} \partial_{J} \ln H_{I}-\partial_{J} H_{(P, p)} \partial_{I} \ln H_{J}=0 \\
& \quad P \neq I, J, \quad p=1, \ldots, n_{P}, \\
& \partial_{I J} H_{(J, j)}-\partial_{I} H_{(J, \lambda)} \partial_{J} \ln H_{I}-\partial_{J} H_{(J, j)} \partial_{I} \ln H_{J} \\
& \quad=H_{(J, j-1)} \partial_{I} \ln H_{J}-\partial_{I} H_{(J, j-1)}, \\
& \quad j=1, \ldots, n_{J} .
\end{aligned}
$$

Here $I \neq J, H_{(J, 0)} \equiv 0$. In terms of the linear operators

$$
\begin{aligned}
& A_{I J}=\partial_{I J}-\partial_{J} \ln H_{I} \partial_{I}-\partial_{I} \ln H_{J} \partial_{J}, \\
& B_{I J}=-\partial_{I}+\partial_{I} \ln H_{J}, \quad I \neq J,
\end{aligned}
$$

these conditions can be written as

$$
\begin{aligned}
& A_{I J} H_{(P, p)}=0, \quad P \neq I, J, \\
& A_{I J} H_{(J, J)}=B_{I J} H_{(J, j-1),} H_{(J, 0)}=0, \quad I \neq J .
\end{aligned}
$$

[The possibility that $L$, Eq. (2.8), has an additive term of the form $H(x) u$ can be treated as a special case of our considerations. Formally Eqs. (2.10) are still the separation conditions for $L=E$ where now $H_{(J, 0)}=H, H_{(J,-1)}=0$, $J=1, \ldots, N$, and the index $j$ takes the values $\left.0,1, \ldots, n_{J}.\right]$

Similarly, the integrability conditions $(2.5)$ for the homogeneous equation $L=0$ take the form

$$
\begin{aligned}
& A_{I J} H_{(P, p)}=Q_{I J}(x) H_{(P, P)}, \quad P \neq I, J, \\
& A_{I J} H_{(J, j)}=B_{I J} H_{(J, j-1)}+Q_{I J}(x) H_{(J, \lambda)}, \\
& H_{(J, 0)}=0, \quad I \neq J,
\end{aligned}
$$

where $Q_{I J}$ is a function of the independent variables $x_{K}$ alone. ed.

The two sets of integrability conditions are closely relat-

Lemma 1: If the functions $\left\{H_{(I, i)}\right\}$ satisfy $(2.12)$ and $R(x)$ is nonzero then the functions $\left\{H_{(I, I)}^{\prime}=R H_{(I, i)}\right\}$ satisfy (2.13). Indeed

$$
\begin{aligned}
-Q_{I J}= & 2 \partial_{I} \ln R \partial_{J} \ln R \\
& +\partial_{I} \ln R \partial_{J} \ln H_{I}+\partial_{J} \ln R \partial_{I} \ln H_{J} .
\end{aligned}
$$

Suppose the functions $\left\{H_{(I, i)}\right\}, I=1, \ldots, N, i=1, \ldots, n_{I}$ are not all zero and set $H_{I, 0}=0$. Then there must exist some $H_{(K, k)} \neq 0$ such that $H_{(K, k-1)}=0$. Let $H_{(I, i)}^{\prime}=H_{(I, i)} / H_{(K, k)}$.

Lemma 2: The functions $\left\{H_{(I, i)}\right\}$ satisfy conditions (2.13) if and only if the functions $\left\{H_{(I, i)}^{\prime}\right\}$ satisfy (2.12), i.e.,

$$
\begin{aligned}
& \partial_{I J} H^{\prime}{ }_{(P, p)}-\partial_{J} \ln H_{I}^{\prime} \partial_{I} H^{\prime}{ }_{(P, p)}-\partial_{I} \ln H^{\prime}{ }_{J} \partial_{J} H^{\prime}{ }_{(P, p)}=0 \text {, } \\
& P \neq I, J, \\
& \partial_{I J} H^{\prime}{ }_{(J, \lambda)}-\partial_{J} \ln H_{I}^{\prime} \partial_{I} H^{\prime}{ }_{(J, \lambda)}-\partial_{I} \ln H_{J}^{\prime} \partial_{J} H_{(J, \lambda)}^{\prime} \\
& =H_{(J, j-1)}^{\prime} \partial_{I} \ln H^{\prime}{ }_{J}-\partial_{I} H^{\prime}{ }_{(J, j-1)} \text {, } \\
& \text { for } I \neq J \text {. }
\end{aligned}
$$

It follows from these lemmas that all sets of functions $\left\{H_{(I, i)}\right\}$ satisfying conditions (2.13) are of the form $H_{(I, i)}=R H_{(I, i)}^{\prime}$, where the $\left\{H_{(I, i)}^{\prime}\right\}$ satisfy conditions (2.12). In the next section we will show how to find all solutions of Eqs. (2.12).

\section{D-STÄCKEL MATRICES}

Consider a coordinate set $x_{1}, \ldots, x_{N}$ and let $n_{1}, \ldots, n_{N}$ be positive integers with $n=\sum_{I=1}^{n} n_{I}$. Let $S=\left(S_{(I, i), l}\left(x_{I}\right)\right)$ be an $n \times n$ matrix with the properties following.

$$
\begin{aligned}
& \text { (1) } S_{(I, i), l}\left(x_{I}\right)=\frac{d^{i-1}}{d x_{I}{ }^{i-1}} S_{(I, 1), l}\left(x_{I}\right), \\
& i=1,2, \ldots, n_{I} .
\end{aligned}
$$

[Here, the rows of $S$ are designated by the index $(I, i)$, where $I=1, \ldots, N, i=1, \ldots, n_{I}$. The columns of $S$ are designated by the index $l=1,2, \ldots, n$. Thus row $(I, i)$ depends only on the variable $x_{I}$ and is the $i-1$ derivative of row $(x, 1)$.]

(2) $\operatorname{det} S \neq 0$.

(3) $T^{1,(J, \lambda)} \neq 0, J=1, \ldots, N, h=1, \ldots, n_{J}$, where $T=S^{-1}$, i.e., 


$$
\sum_{l=1}^{n} S_{(I, \eta, I}\left(x_{I}\right) T^{l,(J, j)}=\delta_{(I, i)}^{(J, j)}
$$

We say that a matrix $S$ satisfying properties (1)-(3) is a differential-Stäckel matrix (D-Stäckel matrix). If $n_{1}=\cdots=n_{N}=1$ then $S$ is simply the usual Stäckel matrix. ${ }^{2,3}$ In order to obtain results about D-Stäckel matrices that are useful for separation of variables we need to characterize the inverse matrix $T$. For this we generalize Eisenhart's study of Stäckel matrices. ${ }^{3.6}$

Differentiating (3.2) with respect to $x_{I}$, we obtain

$$
\sum_{l} S_{(I, i+1), l}\left(x_{I}\right) T^{l,(J, j)}+\sum_{l} S_{(I, I), l} \partial_{x_{I}} T^{l,(J, j)}=0
$$

where we adopt the convention $S_{\left(I, n_{I}+1\right), l}=0$. Since $S$ is nonsingular it follows that

$$
\partial_{x_{I}} T^{l,(J, \hat{\jmath})}=f_{I}^{(J, j)} T^{l,\left(I, n_{l}\right)}-T^{l,(J, j-1)} \delta_{I}^{J},
$$

where $f_{I}^{(J, j)}$ is a function and we adopt the convention $T^{l,(J, 0)}=0$.

$$
\begin{aligned}
& \text { Now set } \\
& T^{l,(J, \lambda)}=\rho_{(J, \lambda)}^{l} H_{(J, \lambda)}, \quad \rho_{(J, \lambda)}^{1}=1 .
\end{aligned}
$$

In particular, $T^{1,(J, \lambda)}=H_{(J, j)}$. We will characterize $T$ in terms of the "roots" $\rho_{(J, j)}^{l}$ and the $H_{(J, j)}$. Substituting (3.5) in (3.4) we obtain

$$
\begin{aligned}
& \partial_{I} \rho_{(J, j)}^{l} H_{(J, j)}+\rho_{(J, j)}^{l} \partial_{I} H_{(J, j)} \\
& \quad=f_{I}^{(J, n)} \rho_{\left(I, n_{j}\right)}^{l} H_{\left(I, n_{I}\right)}-\rho_{(J, j-1)}^{l} H_{(J, j-1)} \delta_{I}^{J},
\end{aligned}
$$

where $\rho_{(J, 0)}^{l}=H_{(J, 0)}=0$. For $l=1$, Eq. (3.6) reduces to

$$
\partial_{I} H_{(J, j)}=f_{I}^{(J, j)} H_{\left(I, n_{I}\right)}-H_{(J, j-1)} \delta_{I}^{J},
$$

in view of (3.5). Solving this expression for $f_{I}^{(J, j)} H_{\left(I, n_{l}\right)}$ and substituting into (3.6) we obtain the desired characterization

$$
\begin{aligned}
\partial_{I} \rho_{(J, j)}^{l}= & \left.\rho_{\left(I, n_{J}\right)}^{l}-\rho_{(J, j)}^{l}\right) \partial_{I} \ln H_{(J, \lambda)} \\
& +\left(\rho_{\left(I, n_{\lambda}\right)}^{l}-\rho_{(I, j-1)}^{l}\right) \frac{H_{(J, j-1)}}{H_{(J, j)}} \delta_{I}^{J},
\end{aligned}
$$

$I, J=1, \ldots, N, h=1, \ldots, n_{J}$.

At this point we have shown that if $S$ is a D-Stäckel matrix then the system of equations

$$
\begin{aligned}
\partial_{I} \rho_{(J, j)}= & \left(\rho_{\left(I, n_{I}\right)}-\rho_{(J, j)}\right) \partial_{I} \ln H_{(J, \lambda)} \\
& +\left(\rho_{\left(I, n_{I}\right)}-\rho_{(J, j-1)}\right) \frac{H_{(J, j-1)}}{H_{(J, j)}} \delta_{I}^{J},
\end{aligned}
$$

$I, J=1, \ldots, N, h=1, \ldots, n_{J}$, where $H_{(J, \lambda)}=T^{1,(J, \lambda)}$ admits a full linearly independent set of $n$ vector-valued solutions $\left\{\rho_{(J, j)}^{l}\right\}, l=1, \ldots, n$.

Conversely, suppose we are given a set of $n$ nonzero functions $\left\{\boldsymbol{H}_{(J, j)}\right\}$ such that the system (3.9) admits a full linearly independent set of $n$ vector-valued solutions $\left\{\rho_{(J, j)}^{l}\right\}$. Since $\rho_{(J, j)} \equiv 1$, all $J, j$, is a solution, without loss of generality we can include it in our basis set and assume $\rho_{(J, n)}^{1}=1$. It follows that the $n \times n$ matrix $T$ defined by $(3.5)$ is invertible. Let $S=T^{-1}$, i.e.,

$$
\sum_{l=1}^{n} S_{(I, i), l} T^{l,(J, j)}=\delta_{(I, i)}^{(J, j)}
$$

It follows from (3.9) and (3.5) that (3.4) holds with

$$
f_{I}^{(J, j)}=H_{\left(I, n_{j}\right)}^{-1}\left(\partial_{I} H_{(J, j)}+H_{(J, j-1)} \delta_{I}^{J}\right) .
$$

Differentiating both sides of (3.10) with respect to $x_{K}$ and using (3.4), we find

$$
\sum_{l=1}^{n} \partial_{K} S_{(I, i), l} T^{l,(J, j)}=\delta_{K}^{J} \delta_{(I, j)}^{(J, j-1)}-f_{K}^{(J, \lambda)} \delta_{(I, i)}^{\left(K, n_{K}\right)}
$$

It follows that $\partial_{K} S_{(I, i), l}=0$ if $K \neq I$ and $\partial_{I} S_{(I, i), l}=S_{(I, i+1), l}$ for $i=1, \ldots, n_{I}-1$. Thus $S$ is a D-Stäckel matrix.

Theorem 1: Let $\left\{H_{(J, \lambda)}\right\} \quad\left(J=1, \ldots, N, j=1, \ldots n_{J}\right.$, $\left.\Sigma_{J} n_{J}=n\right)$ be a set of $n$ nonzero functions of the $N$ variables $x_{I}$. There exists an $n \times n$ D-Stäckel matrix $S=\left(S_{(I, I), l}\left(x_{I}\right)\right)$ with inverse $T=\left(T^{l,(J, j)}\right)$ such that $H_{(J, j)}=T^{1,(J, j)}$ if and only if $\left\{H_{J, D}\right\}$ satisfies Eqs. (2.10a) and (2.10b).

Proof: It is straightforward to verify that (2.10a) and $(2.10 \mathrm{~b})$ are simply the integrability conditions $\partial_{K}\left(\partial_{I} \rho_{(J, \lambda)}\right)=\partial_{I}\left(\partial_{K} \rho_{(J, \lambda)}\right), K \neq I$, for the system (3.9). Thus if $\left\{H_{(J, j)}\right\}$ satisfies the integrability conditions then $(3.9)$ has $n$ independent vector-valued solutions and we can construct a D-Stäckel matrix $S$ such that $H_{(J, j)}=T^{1,(J, \lambda)}$.

Conversely, if $H_{(J, j)}=T^{1,(J, j)}$ and $T^{-1}=S$ for some DStäckel matrix $S$ then the system (3.9) admits $n$ independent vector-valued solutions and the integrability conditions (2.10) must be satisfied.

We now have a partial answer to the problems posed in Sec. II. Consider the equation $L=E$ in $N$ independent variables $\mathrm{x}_{l}$, where

$$
L=\sum_{J=1}^{N} \sum_{j=1}^{n_{J}} H_{\langle J, j)}(x) u_{J, j},
$$

and suppose each of the $H_{(J, \lambda)}$ is nonzero. This equation admits (regular) additively separable solutions provided the conditions (2.10) are satisfied. These conditions imply the existence of a D-Stäckel matrix $S$ such that $H_{(J, j)}=T^{1,(J, j)}$. The separation equations are evident

$$
\begin{aligned}
& u_{J, j}+\sum_{l=1}^{n} S_{(J, j), l}\left(x_{J}\right) \lambda_{l}=0, \\
& 1 \leqslant J \leqslant N, \quad 1 \leqslant j \leqslant n_{J}, \quad \lambda_{1}=-E .
\end{aligned}
$$

Here there are $n$ separation parameters $\lambda_{l}$. The separable solutions $u$ are obtained by integrating the $N$ first-order ordinary differential equations

$$
u_{J, 1}+\sum_{l=1}^{n} S_{(J, 1), l}\left(x_{J}\right) \lambda_{l}=0 .
$$

The remaining $n-N$ equations are redundant since they are obtained by differentiating the basic set (3.13). The number of parameters in the solution $u$ is $n+\Sigma_{J} n_{J}+1$, in agreement with the prediction in Sec. II. Multiplying the separation equation (3.12) for $u_{J, j}$ by $T^{1,(J, \lambda)}$ and summing over the index $(J, j)$ we once again obtain $L=E$ for $E=-\lambda_{1}$.

The treatment for the equation $L=0$ is similar. Suppose

$$
L=\sum_{J=1}^{N} \sum_{j=1}^{n_{J}} H_{(J, j)}^{\prime}(x) u_{J, j},
$$

where none of the $H_{(J, j)}^{\prime}$ is zero and suppose these functions satisfy the separability conditions (2.14). Then there is a nonzero function $R(x)$ such that $H_{(J, n)}^{\prime}=R H_{(J, \lambda)}$, where the $H_{(J, j)}$ satisfy conditions (2.10) and, thus, determine a DStäckel matrix $S$. The separation equations are (3.12) with $\lambda_{1}=0$. There are $n-1$ separation parameters and a sepa- 
rated solution contains $n=\Sigma_{J} n_{J}$ parameters. Multiplying the separation equation (3.12) for $u_{J, j}$ by $R T^{1,(J, n)}$ and summing over the index $(J, j)$, we rederive $L=0$, since $\lambda_{1}=0$.

\section{ANALYSIS OF THE SEPARATION EQUATIONS}

We do not as yet have a complete solution of the integrability conditions characterizing regular separation for the linear equation $L=E$

$$
\begin{aligned}
& A_{I J} H_{(P, p)}=0, \quad P \neq I, J, \\
& A_{I J} H_{(J, J)}=B_{I J} H_{(J, j-1)}, \quad H_{(J, 0)}=0, \quad I \neq J,
\end{aligned}
$$

where

$$
\begin{aligned}
& A_{I J}=\partial_{I J}-\partial_{J} \ln H_{I} \partial_{I}-\partial_{I} \ln H_{J} \partial_{J}, \\
& B_{I J}=-\partial_{I}+\partial_{I} \ln H_{J}, \quad H_{J}=H_{\left(J, n_{J}\right)} \neq 0,
\end{aligned}
$$

$$
1 \leqslant J \leqslant N, \quad 1 \leqslant j \leqslant n_{J}, \quad \sum_{J} n_{J}=n .
$$

In order that Theorem 1 can be applied to obtain a D-Stäckel matrix we must have all $H_{(J, j)} \neq 0$. However, we are assuming only that $H_{J} \neq 0$. Furthermore, it is easy to construct examples of separable systems where at least one of the $H_{(J, J)}$ vanishes.

A more detailed analysis of the structure of Eqs. (4.1) will resolve the difficulty. Suppose we are given $N$ nonzero functions $H_{J}$ satisfying $A_{I J} H_{P}=0$ for $P \neq I, J, I \neq J$. Our task will be to construct a finite set of functions $H_{(J, \lambda)}$ with $H_{\left(J, n_{j}\right)}=H_{J}$ such that Eqs. (4.1) are satisfied. (We do not require that the $H_{(J, j)}$ are all nonzero.) Initially we will not know the values of the integers $n_{J}$.

The construction process is based on the second equation of $(4.1)$, which we can write in the form

$$
\partial_{I}\left[H_{(K, k-1)} / H_{K}\right]=\left(\partial_{K} \ln H_{I} \partial_{I} H_{(K, k)}+\partial_{I} \ln H_{K} \partial_{K} H_{(K, k)}-\partial_{I K} H_{(K, k)}\right) / H_{K}, \quad I \neq K
$$

If $H_{(K, k)}$ is known then we can construct $H_{(K, k-1)}$ from (4.3) by quadrature.

Lemma 5: Suppose the $N$ nonzero functions $H_{P}$ satisfy $A_{I J} H_{P}=0$ for $P \neq I, J, I \neq J$ and suppose the function $H_{(K, k)}($ fixed $K, k)$ satisfies $A_{I J} H_{(K, k)}=0, K \neq I, J, I \neq J$. Then the $N-1$ equations (4.3) are compatible and have the general solution

$$
H_{(K, k-1)}=\widetilde{H}_{(K, k-1)}+f^{(k-1)}\left(x_{K}\right) H_{K},
$$

where $\widetilde{H}_{(K, k-1)}$ is a particular solution and $f^{(k-1)}$ is an arbitrary function of $x_{K}$. The solution satisfies

$$
A_{I J} H_{(K, k-1)}=0, \quad K \neq I, J, \quad I \neq J .
$$

Proof: The compatibility requirement $\partial_{J}\left(\partial_{I}\left(H_{(K, k-1)} / H_{K}\right)\right)=\partial_{I}\left(\partial_{J}\left(H_{(K, k-1)} / H_{K}\right)\right), \quad I, J \neq K$ and (4.5) are straightforward consequences of (4.3) and the conditions $A_{I J} H_{P}=0, A_{I J} H_{(K, k)}=0$.

It follows from Lemma 5 that for each $K$ we can always construct functions $H_{(K, k-1)}$ through a step-by-step procedure using the second of Eqs. (4.1), such that the first of Eqs. (4.1) is automatically satisfied. At each step the solution $H_{(K, k-1)}$ is arbitrary up to the additive term $f^{(k-1)}\left(x_{K}\right) H_{K}$ and we simply choose one of these solutions. Thus we generate an infinite sequence $\left\{H_{(K, k)}=H_{K}^{(I)}\right\}, l=0,1,2, \ldots$, where $n_{K-l}=k$ (but $n_{K}$ is unknown)

$$
A_{I K} H_{K}^{(l)}=B_{I K} H_{K}^{(l+1)}, \quad I \neq K, \quad H_{K}=H_{K}^{(0)}
$$

The following properties of the operators $A_{I K}, B_{I K}$ will prove useful:

$$
\begin{aligned}
& B_{I K} F(x)=0, \text { for all } I \neq K \\
& \quad \Leftrightarrow F(x)=f\left(x_{K}\right) H_{K}, \\
& A_{I K}\left(f\left(x_{K}\right) H_{K}^{(l)}\right)=B_{I K}\left(f H_{K}^{(l+1)}-f^{\prime} H_{K}^{(l)}\right),
\end{aligned}
$$

where $f^{\prime}=\partial_{K} f$.

Suppose there is a smallest finite positive integer $m_{K}$ for which functions $f_{(i)}\left(x_{K}\right)$ exist such that

$$
H_{K}^{\left(m_{K}\right)}=\sum_{i=0}^{m_{K}-1} f_{(i)}\left(x_{K}\right) H_{K}^{(i)} .
$$

Lemma 6: $\operatorname{Each} H_{K}^{\left(m_{K}+s\right)}, s=0,1,2, \ldots$, is a linear condition of the finite set $\left\{H_{K}^{(l)}: l=0, \ldots, m_{K}-1\right\}$ with coefficients that are functions of $x_{K}$.

Proof: The proof is by induction on $s$. The statement is clearly true for $s=0$. We assume it holds for $s=t$

$$
H_{K}^{\left(m_{K}+t\right)}=\sum_{i=0}^{m_{K}-1} g_{(i)}\left(x_{K}\right) H_{K}^{(i)} .
$$

Now

$$
\begin{aligned}
B_{I K} H_{K}^{\left(m_{K}+t-1\right)} & =A_{I K} H_{K}^{\left(m_{K}+t\right)}=A_{I K}\left(\sum_{0}^{m_{K}-1} g_{(i)} H_{K}^{(i)}\right) \\
& =B_{I K}\left(\sum_{0}^{m_{K}-1} g_{(i)} H_{K}^{(i+1)}-\sum_{0}^{m_{K}-1} g_{(i)}^{\prime} H_{K}^{(i)}\right) .
\end{aligned}
$$

Hence, by (4.7) there is a function $g\left(x_{K}\right)$ such that

$$
\begin{aligned}
& H_{K}^{\left(m_{K}+t+1\right)}=\sum_{i=0}^{m_{K}-1} h_{(i)}\left(x_{K}\right) H_{K}^{(i)}, \\
& h_{(i)}\left(x_{K}\right)= \begin{cases}g_{(i-1)}-g_{(i)}^{\prime}, & 1 \leqslant i \leqslant m_{K}-1, \\
g-g_{(0)}^{\prime}, & i=0 .\end{cases}
\end{aligned}
$$

Let $\left\{\mathscr{H}_{K}^{(l)}\right\},\left\{h_{K}^{(l)}\right\}, l=0,1,2, \ldots$, be two sequences constructed by the procedure (4.6).

Lemma 7: There is a sequence of functions $g_{1}\left(x_{K}\right), g_{2}\left(x_{K}\right), \ldots$, and expressions $L_{i, j}\left(g_{1}, g_{2}, \ldots, g_{i-j-1}\right)$ with $L_{i 0}=0, L_{i, i-1}=0$, and $L_{i+1, j}=L_{i, j-1}+g_{i-j}^{\prime}-L_{i, j}^{\prime}$ such that

$$
\begin{aligned}
\mathscr{H}_{K}^{(i)} & =h_{K}^{(i)}+\sum_{j=0}^{i-1}\left(g_{i-j}\left(x_{K}\right)-L_{i, j}\left(x_{K}\right)\right) h_{K}^{(j)}, \\
i & =0,1,2, \ldots .
\end{aligned}
$$

Any such sequence $\left\{g_{l}\left(x_{K}\right)\right\}$ together with $\left\{h_{K}^{(I)}\right\}$ determines a new sequence of solutions $\left\{\mathscr{H}_{K}^{(i)}\right\}$ of (4.6). The induction 
proof of this result is similar to that of the preceding lemma.

Now let $\left\{H_{K}^{(l)}\right\}$ be the solution sequence treated in Lemma 6 and consider the relation (4.9). Set $h_{K}^{(i)}=H_{K}^{(i)}$ in (4.10) and choose $g_{1}, \ldots, g_{m_{K}-1}$ recursively such that

$$
-f_{(j)}=g_{m_{K}-j}-L_{m_{K}, j}, \quad j=0,1, \ldots, m_{K}-1 .
$$

Then $\mathscr{H}_{K}^{\left(m_{K}\right)}=0$. We see that there is a solution sequence $\left\{\mathscr{H}_{K}^{(l)}\right\}$ with $\mathscr{H}_{K}^{(0)}, \ldots, \mathscr{H}_{K}^{\left(m_{K}-1\right)}$ nonzero and all further terms zero. According to Lemma 7 all other solution sequences are linear combinations of these $m_{K}$ nonzero terms.

Lemma 8: The integer $m_{K}$, if it exists, is unique.

In general, there is no finite integer $m_{K}$ for which (4.9) holds. [An example is $N=2, H_{1}=1, H_{2}=\exp \left(x_{1} x_{2}\right)$. Here $m_{1}=1$, but $m_{2}$ does not exist.] However, if the $H_{(J, j)}$ satisfy equations (4.1), i.e., if they correspond to a regular separable system for the equation $L=E$ then the integers $m_{J}$ always exist and $1 \leqslant m_{J} \leqslant n_{J}$. Thus there is a set of $\Sigma_{J=1}^{N} m_{J}$ functions $\left\{h_{(J, \lambda)}\right\}, 1 \leqslant j \leqslant m_{J}$ satisfying (4.1) such that $H_{J}=h_{\left(J, m_{J}\right)}$ and each $h_{(J, D)}$ is nonzero. Using Lemma 7 we can express the equation $L=E$ in terms of the new functions $h_{(J, N)}$.

Lemma 9:

$$
L=\sum_{K=1}^{N} \sum_{k=1}^{n_{K}} H_{(K, k)} u_{K, k}=\sum_{K=1}^{N} \sum_{k=1}^{m_{K}} h_{(K, k)} U_{K, k},
$$

where

$$
\begin{aligned}
U_{K, k}\left(u_{k, l}, x_{K}\right)= & u_{K, n_{K}-m_{K}+k} \\
& +\sum_{s=1}^{n_{K}-m_{K}+k-1}\left(g_{n_{K}-m_{K}-s+k}\left(x_{K}\right)\right. \\
& \left.-L_{n_{K}-s, m_{K}-k}\left(x_{K}\right)\right) u_{K, s} .
\end{aligned}
$$

In particular,

$$
\partial_{K} U_{K, k}=U_{K, k+1}, \quad 1 \leqslant k \leqslant m_{K}-1 .
$$

It follows from this result and Theorem 1 that when $L=E$ is separable then there exists a set of $m=\Sigma_{J=1}^{N} m_{J}$ nonzero functions $h_{(J, j)}$ and an associated $m \times m$ D-Stäckel matrix $S$ such that $h_{(J, j)}=T^{1,(J, \lambda)}$, where $T=S^{-1}$ and the separation equations for $L=E$ take the form

$$
\begin{array}{cc}
U_{K, k}+\sum_{l=1}^{m} S_{(K, k), l}\left(x_{K}\right) \lambda_{l}=0, & K=1, \ldots, N, \\
1 \leqslant K \leqslant N, \quad 1 \leqslant k \leqslant m_{K} \leqslant n_{K}, & \lambda_{1}=-E .
\end{array}
$$

There are $m$ separation parameters $\lambda_{l}$. The separable solutions $u$ are determined by solving the $N$ ordinary differential equations

$$
U_{K, 1}+\sum_{l=1}^{m} S_{(K, 1), I}\left(x_{K}\right) \lambda_{l}=0 .
$$

The remaining $m-N$ equations (4.11) are redundant, since they are obtained by differentiating the basic set (4.12). The highest derivative term in $U_{K, 1}$ is $u_{K, n_{K}-m_{K}+1}$ so each equation (4.12) is of order $n_{K}-m_{K}+1$. The number of parameters in the solution $u$ is $m+\Sigma_{K}\left(n_{K}-m_{K}\right)+1=n+1$. We now have the complete solution of the separation of Eqs. (2.10).

\section{SEPARATION OF LAPLACE EQUATIONS}

Suppose $\Delta_{N}$ is the Laplace-Beltrami operator on a local pseudo-Riemannian manifold $V^{N}$. In local orthogonal coordinates $x_{I}$ we have

$$
\Delta_{N}=\frac{1}{h} \sum_{I=1}^{N} \partial_{I}\left(h H_{I} \partial_{I}\right)
$$

where

$$
d s^{2}=\sum_{I=1}^{N} H_{I}^{-1} d x_{I}^{2}, \quad h^{2}=\prod_{I} H_{I}^{-1} .
$$

It is of interest to determine the relationships between the well-developed theory of multiplicative $R$ separation for the Laplace equation $\Delta_{N} u=0$ (Refs. 7-9) and additive separation. Recall that multiplicative $R$ separation in the orthogonal coordinates $x_{I}$ leads to solutions for the Laplace equation of the form

$$
u=e^{R(x)} \prod_{I=1}^{N} u^{(I)}\left(x_{I}\right)
$$

where the fixed function $R$ is independent of the separation parameters. Similarly we can introduce additive $R$ separation

$$
u=e^{R(x)}\left(\sum_{I=1}^{N} u^{(I)}\left(x_{I}\right)\right) .
$$

The following is a straightforward consequence of the principal results of this paper.

Theorem 2: If the Laplace equation $\Delta_{N} U=0$ is multiplicatively $R$ separable in the orthogonal coordinates $x_{I}$ then it is additively $R$ separable in these same coordinates if and only if $e^{-R} \Delta_{N} e^{R}=c H_{I}, I=1, \ldots, N$, where $c$ is a constant.

In each of these cases a true Stäckel matrix determines the separation; no nontrivial D-Stäckel matrices appear. Note that true multiplicative separation $(R \equiv 1)$ always leads to additive separation.

On the other hand, Laplace equations may admit additive separation in an orthogonal coordinate system for which no multiplicative $R$ separation is possible. For example, consider the three-dimensional manifold with metric coefficients

$$
H_{1}=H_{2}=\left(x_{1}+x_{2}\right)^{5}, \quad H_{3}=\left(x_{1}+x_{2}\right)^{4} .
$$

Then

$\Delta_{3}=\left(x_{1}+x_{2}\right)^{4}\left[\left(x_{1}+x_{2}\right)\left(\partial_{11}+\partial_{22}\right)-2\left(\partial_{1}+\partial_{2}\right)+\partial_{33}\right]$,

and since (5.4) is not conformal to a Stäckel form metric, no multiplicative $R$ separation is possible. However, since

$$
\begin{aligned}
\left(x_{1}+x_{4}\right)^{-4} \Delta_{3} u= & \left(x_{1}+x_{2}\right)\left(u_{1,2}+u_{2,2}\right) \\
& -2\left(u_{1,1}+u_{2,1}\right)+u_{3,2}=0,
\end{aligned}
$$

we have

$$
\begin{aligned}
& H_{1}^{\prime}=H_{2}^{\prime}=x_{1}+x_{2}, \quad H_{3}^{\prime}=1, \\
& H_{(1,1)}^{\prime}=H_{(2,1)}^{\prime}=-2,
\end{aligned}
$$

which satisfies Eqs. (2.10) [or (2.14)] with $n_{1}=n_{2}=2$, $n_{3}=1$. Thus the Laplace equation admits additive separation in these coordinates corresponding to a $5 \times 5 \mathrm{D}$-Stäckel matrix.

\section{ACKNOWLEDGMENT}

This work has been partially supported by National Science Foundation Grant No. MCS 82-19847. 
${ }^{1}$ E. G. Kalnins and W. Miller, "Intrinsic characterization of variable separation for the partial differential equations of mechanics," in Proceedings of IUTAM-ISIMM Symposium on Modern Development in Analytical Mechanics, Turin, 1982 (Atti della Academia delle Scienze di Torino, Turin, 1983), Supplement to Vol. 117, pp. 511-533.

${ }^{2}$ P. Stäckel, Über die Integration der Hamilton-Jacobischen Differentialgleichung Mittels Separation der Variabelen (Habitilatationsschrift, Halle, 1891).

${ }^{3}$ L. P. Eisenhart, Ann. Math. 35, 284 (1934).
${ }^{4}$ T. Levi-Civita, Math. Ann. 59, 383 (1904).

${ }^{5} \mathrm{~T}$. W. Koornwinder, "A precise definition of separation of variables," in Lecture Notes in Mathematics, Vol. 810 (Springer, Berlin, 1980).

${ }^{6}$ L. P. Eisenhart, Riemannian Geometry (Princeton U. P., Princeton, 1949), Appendix 13.

${ }^{7}$ P. Moon and D. E. Spencer, J. Franklin Inst. 253, 585 (1952).

${ }^{8}$ V. N. Shapovalov, Differential Equations 16, 1864 (1980); 10, 1212 (1981).

${ }^{9}$ E. G. Kalnins and W. Miller, Trans. Am. Math. Soc. 244, 241 (1978). 